\title{
The Fermion Determinant in Two Dimensional Minkowski Space: Zeros and Related Properties ${ }^{\star}$
}

\author{
D. Cangemi, M. Makowka and G. Wanders

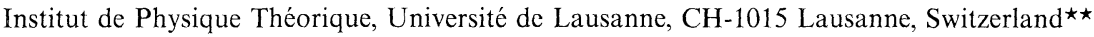

\begin{abstract}
We present a detailed analysis of the non-abelian determinant of massless fermions in two dimensional Minkowski space. In the framework of the external field problem, the determinant vanishes if the out-and ingoing vacua are orthogonal; the gauge potentials for which this happens are identified. Causality implies that the effective action obtained from the sum of fermion loops has the right singularity at a zero of the determinant. Such a zero can be reached by a continuous deformation of a potential with non-vanishing determinant. The set of zeros exhibits a rich structure.
\end{abstract}

\section{Introduction and Statement of Results}

It has been realized some time ago that the determinant of massless fermion fields coupled to an external non-abelian gauge potential in a two dimensional space can be determined explicitly [1]. In a previous work [2] which will be referred to as I, we derived the effective action, that is the logarithm of the determinant, in the framework of $1+1$ dimensional Minkowski space-time.

At first sight our effective action, as well as the action obtained in a euclidean space, seems to be finite for all reasonable potentials. If this would be the case, the fermion determinant would never vanish. On the other hand, one knows that the determinant in a compactified euclidean space vanishes whenever the external potential allows zero modes of the fermion fields [3]. As nothing seems to prevent the occurrence of zero modes in two dimensions, there is a problem, at least in the euclidean setting, which has been addressed by Kupiainen and Mickelsson [4]. They find that there are potentials for which the fermion fields have zero modes and to which the known formula of the effective action does not apply. The main motivation of the present work was to find out if the Minkowski space determinant too has zeros and how they are compatible with our expression of the effective

\footnotetext{
* Work partially supported by the Swiss National Science Foundation

$\star \star$ Postal address: Bâtiment des Sciences physiques, Université de Lausanne, CH-1015 Lausanne, Switzerland
} 
action. Whereas the possibility of such zeros has been recognized [5], their occurrence has not been investigated until now, as far as we know.

Independently of the existence of zeros of the determinant, there is another reason justifying a closer look at our effective action. This quantity has been defined in I by its perturbation expansion, i.e. as the sum of closed fermion loops. In spite of this, our result is non-perturbative because its derivation involves the conversion of a recurrence relation between successive terms of a perturbation series into an integral equation for their sum. It is the solution of that equation which determines our effective action. If our formula makes sense for potentials for which the sum of loops diverges, it defines a continuation of the effective action outside the domain of convergence of the perturbation expansion. One would like to be sure that this continuation is the correct one. These questions have not been discussed in I.

If we want to check our effective action, we need a source of information on the fermion determinant independent of the fermion loops. We shall resort to the solution of the external field problem of our fermion fields coupled to the given gauge potential $A$. In this context, the determinant det $D[A]$ of the Dirac operator $\not D[A]$ is defined as the vacuum-to-vacuum amplitude $\left(\Omega_{\text {out }}, \Omega_{\text {in }}\right)$, where $\Omega_{\text {in }}$ and $\Omega_{\text {out }}$ are the incoming and outgoing vacua. One gets the modulus of the vacuum amplitude and, from this, the imaginary part of the effective action $W[A](W[A]=$ $(1 / i) \log \operatorname{det} \not D[A])$. The external field problem will be solved in Sect. 2 and it will turn out that there are potentials for which the determinant vanishes. Physically, this comes from the fact that the configuration of outgoing fermion-antifermion pairs created by the potential in the incoming vacuum can be such that $\Omega_{\text {in }}$ is orthogonal to $\Omega_{\text {out }}$.

The existence of zeros of the determinant being firmly established, one has to ask how this agrees with our effective action. A specific feature of the Minkowski space approach is that the effective action is constructed with the help of two causal matrix functions $T_{ \pm}$. They are the solutions of the differential equations

$$
\partial_{ \pm} T_{\mp}(\mathbf{x})=i g A_{ \pm}(\mathbf{x}) T_{\mp}(\mathbf{x})
$$

fulfilling Feynman-Stueckelberg causal boundary conditions. In (1.1) light-cone variables $x^{ \pm}=(1 / \sqrt{2})\left(x^{0} \pm x^{1}\right)$ and light-cone components $A_{ \pm}=(1 / \sqrt{2})\left(A_{0} \pm A_{1}\right)$ are used; $\mathbf{x}$ designates the space-time point specified by $\left(x^{0}, x^{1}\right)$ or $\left(x^{+}, x^{-}\right)$. The question of the existence of causal solutions of Eqs. (1.1) has been dealt with only superficially in I. A complete analysis is presented in Sect. 3; it gives the clue to the compatibility of our formula for the effective action and the external field problem results. One discovers that there are potentials for which the differential equations (1.1) have no solutions verifying the causal boundary conditions. These are precisely the potentials producing a vanishing determinant according to the external field problem. The main result of Sect. 4 is that our effective action makes sense for all potentials for which the determinant is non-zero. Furthermore, as indicated in Sect. 5, the modulus of $\operatorname{det} D[A]$ computed from the imaginary part of $W[A]$ coincides with the result obtained in Sect. 2. This means that $\operatorname{Im} W[A]$ diverges when $A$ varies and goes to a potential with vanishing determinant. Once the occurrence of zeros of the fermion determinant is recognized, one starts asking how they are distributed in the space of all the potentials we admit. There is no 
simple picture providing an answer to this question. Nevertheless we have collected some indications in Sect. 4 and 5 in the case where the gauge group is $S U(N)$. Let A be the space of our admissible potentials, as it will be defined in the following sections, $\mathbf{A}_{/ /}$the subset with non-vanishing determinant $\left(\Omega_{\text {out }}\right.$ has a component parallel to $\left.\Omega_{\text {in }}\right)$ and $\mathbf{A}$ its complement with respect to $\mathbf{A}\left(\Omega_{\text {in }} \perp \Omega_{\text {out }}\right)$. There is an inner region of $\mathbf{A}$ containing small potentials which is entirely in $\mathbf{A}_{/ /}$. Vanishing determinants show up only for large potentials. It is easier to get a precise insight into $\mathbf{A}_{\perp}$ by means of functionals of the potential which will be called transition matrices. In the topological space of these quantities the image of $\mathbf{A}_{/ /}$is dense and path connected to the unit matrix (the image of a vanishing potential). This means that the potentials in $\mathbf{A}_{\perp}$ are limits of sequences in $\mathbf{A}_{/ /} ; \mathbf{A}_{\perp}$ and $\mathbf{A}_{/ /}$are not disconnected. Furthermore the zeros of the determinant are not isolated points in $\mathbf{A}$ : a potential in $\mathbf{A}_{\perp}$ can be changed continuously without leaving $\mathbf{A}_{\perp}$; this set has in fact a very rich internal structure which is described in Sect. 5.

We have explicit examples illustrating all our results. The simplest one is presented in Appendix A and in a separate note [6].

\section{The External Field Problem}

An $N$-plet of massless Dirac fermion fields $\psi$ belonging to a unitary representation $\rho(G)$ of a compact gauge group $G$ is coupled to an external gauge potential $A$. In $1+1$ dimensions the Dirac equation gives:

$$
\partial_{ \pm} \psi_{\mp}(\mathbf{x})=i g A_{ \pm}(\mathbf{x}) \psi_{\mp}(\mathbf{x}),
$$

where $\psi_{ \pm}$are the spinor components of $\psi$ in the chiral basis $\left(\gamma^{5}=\sigma_{3}\right)$. Introducing an index $\alpha$ with two values, $\alpha= \pm$, the general solution of (2.1) is:

$$
\psi_{\alpha}(\mathbf{x})=R_{\alpha}(\mathbf{x}) \chi_{\alpha}\left(x^{\alpha}\right)
$$

where $\chi$ is an $N$-plet of free canonical massless Dirac fields and $R_{\alpha}$ are $c$-number matrix functions obeying Eqs. (1.1), $R_{\alpha}(\mathbf{x}) \in \rho(G)$.

The case of Weyl fermions is obtained by keeping just one set of the decoupled chiral components, either $\psi_{+}$or $\psi_{-}$.

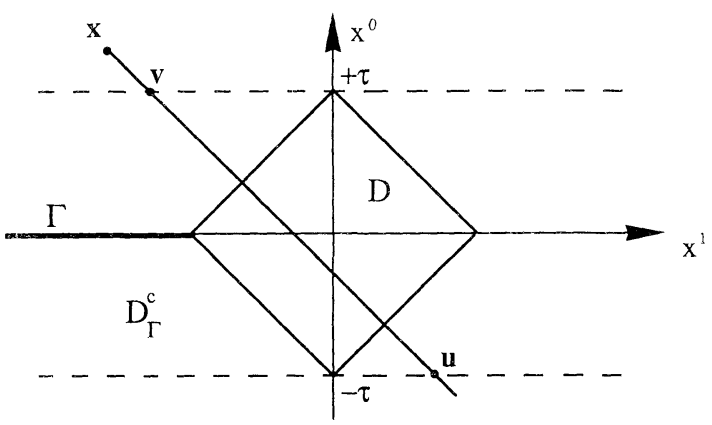

Fig. 1. The localized potential $A$ has its support in the double cone $D$ contained in the strip $\left|x^{0}\right| \leqq \tau$. The gauge function $U$ is single valued in $D_{\Gamma}^{c}$, the complement of $D$ cut along $\Gamma$. The integral in (2.7) is along the straight segment connecting $\mathbf{u}$ and $\mathbf{v}$ 
In this section we restrict ourselves to potentials $A$ whose field strength $F^{\mu v}$ has a compact support located in a double-cone $D$. The potential needs not to be zero in the complement $D^{c}$ of $D$; locally it is a pure gauge there. In general, $A$ will be globally a pure gauge in $D^{c}$ only after this domain has been made simply connected by means of a cut $\Gamma$, as done in Fig. 1 . Then there exists a matrix function $U(\mathbf{x})$ belonging to $\rho(G)$ which is single valued in the cut domain $D_{\Gamma}^{c}$ and such that:

$$
A_{ \pm}(\mathbf{x})=-(i / g) \partial_{ \pm} U(\mathbf{x}) U^{\dagger}(\mathbf{x}) \text { for } \quad x \in D_{I}^{c} .
$$

As $A \neq 0$ in $D^{c}$, the fermion fields don't behave as free in- or outgoing fields for $x^{0} \rightarrow \mp \infty$. Nevertheless the in-out relationship can be described in terms of free and gauge invariant auxiliary fields. Our first goal is to show how this can be done.

We choose the matrices $R_{\alpha}$ as the solutions of (1.1) which coincide with $U$ at time $x^{0}=-\tau$ (see Fig. 1). As a consequence of (1.1) and (2.3), this equality holds also elsewhere, in particular at all previous times:

$$
R_{\alpha}(\mathbf{x})=U(\mathbf{x}) \text { for } \quad x^{0} \leqq-\tau .
$$

If we look at $R_{+}$we have for $x^{0}>-\tau$ :

$$
R_{+}(\mathbf{x})=P \exp \left[i g \int_{u^{-}}^{x-} d y^{-} A_{-}\left(x^{+}, y^{-}\right)\right] U(\mathbf{u}) .
$$

The symbol $P$ means path ordering. The point $\mathbf{u}$ is defined by $u^{0}=-\tau, u^{+}=x^{+}$; we shall also need $\mathbf{v}$ such that $v^{0}=\tau, v^{+}=x^{+}$. For $x^{0}>\tau$, Eq. (2.5) becomes:

with

$$
R_{+}(\mathbf{x})=U(\mathbf{x}) V_{+}\left(x^{+}\right),
$$

$$
V_{+}\left(x^{+}\right)=U^{\dagger}(\mathbf{v}) P \exp \left[i g \int_{u^{-}}^{v^{-}} d y^{-} A_{-}\left(x^{+}, y^{-}\right)\right] U(\mathbf{u}) .
$$

This matrix function and the corresponding $V_{-}\left(x^{-}\right)$will be called transition matrices; they define the in-out relationship and will play a central role.

If we now define in- and out-fields as follows:

$$
\begin{aligned}
& \phi_{\alpha}^{\text {in }}\left(x^{\alpha}\right) \stackrel{\text { dcf }}{=} \chi_{\alpha}\left(x^{\alpha}\right)=\left.U^{\dagger}(\mathbf{x}) \psi_{\alpha}(\mathbf{x})\right|_{x^{0}<-\tau}, \\
& \left.\phi_{\alpha}^{\text {out }}\left(x^{\alpha}\right) \stackrel{\text { def }}{=} U^{\dagger}(\mathbf{x}) \psi_{\alpha}(\mathbf{x})\right|_{x^{0}>\tau}
\end{aligned}
$$

these fields are related by

$$
\phi_{\alpha}^{\text {out }}\left(x^{\alpha}\right)=V_{\alpha}\left(x^{\alpha}\right) \phi_{\alpha}^{\text {in }}\left(x^{\alpha}\right) .
$$

They are canonical free massless Dirac fields: $\phi_{\alpha}^{\text {in }}$ and $\phi_{\alpha}^{\text {out }}$ depend on $x^{\alpha}$ alone and verify canonical anticommutation relations. Furthermore, $V_{\alpha}, \phi_{\alpha}^{\text {in }}$ and $\phi_{\alpha}^{\text {out }}$ are gauge invariant quantities; the gauge dependencies of $\psi_{\alpha}, U$ and $U^{\dagger}$ compensate each other in the definitions (2.7) and (2.8).

The automorphism (2.9) defines the gauge invariant content of the in-out relationship in the framework of the external field problem. It is completely specified 
by the transition matrices $V_{\alpha}$. We notice right away that there is a whole class of potentials leading to one and the same pair of transition matrices. We will come back to this later on.

The automorphism (2.9) is equivalent to a Bogoliubov-Valatin transformation relating the creation and annihilation operators of the in- and out-fields (2.8). If

$$
\phi_{\alpha}^{\substack{\text { in } \\ \text { out }}}\left(x^{\alpha}\right)=(1 / \sqrt{2 \pi}) \int_{0}^{\infty} d k\left[a_{\alpha}^{\substack{\text { in } \\ \text { out }}}(k) e^{-i k x^{\alpha}}+b_{\alpha}^{\text {out }}(k) e^{\imath k x^{\alpha}}\right] \text {, }
$$

(2.9) gives:

$$
\begin{aligned}
a_{\alpha}^{\text {out }}(k) & =\int_{0}^{\infty} \mathrm{d} k^{\prime}\left[K_{1}^{\alpha}\left(k, k^{\prime}\right) a_{\alpha}^{\text {in }}\left(k^{\prime}\right)+K_{2}^{\alpha}\left(k, k^{\prime}\right) b_{\alpha}^{\text {in } \dagger}\left(k^{\prime}\right)\right], \\
b_{\alpha}^{\text {out }}(k) & =\int_{0}^{\infty} d k^{\prime}\left[K_{3}^{\alpha}\left(k, k^{\prime}\right) a_{\alpha}^{\text {in }}\left(k^{\prime}\right)+K_{4}^{\alpha}\left(k, k^{\prime}\right) b_{\alpha}^{\text {in }}\left(k^{\prime}\right)\right] .
\end{aligned}
$$

Notice that $(k / \sqrt{2})$ is the fermion energy.

The operators $K_{n}^{\alpha}$ are obtained from the Fourier transform $\tilde{V}_{\alpha}$ of $\left(V_{\alpha}-1\right)$ :

$$
\tilde{V}_{\alpha}(k)=(1 / 2 \pi) \int d x\left(V_{\alpha}(x)-1\right) e^{-i k x} .
$$

One finds:

$$
\begin{aligned}
& K_{1}^{\alpha}\left(k, k^{\prime}\right)=\delta\left(k-k^{\prime}\right)+\tilde{V}_{\alpha}\left(-k+k^{\prime}\right), \quad K_{2}^{\alpha}\left(k, k^{\prime}\right)=\tilde{V}_{\alpha}\left(-k-k^{\prime}\right) \\
& K_{3}^{\alpha}\left(k, k^{\prime}\right)=\tilde{V}_{\alpha}\left(k+k^{\prime}\right), \quad K_{4}^{\alpha}\left(k, k^{\prime}\right)=\delta\left(k-k^{\prime}\right)+\tilde{V}_{\alpha}\left(k-k^{\prime}\right) .
\end{aligned}
$$

It is well known [7] that the transformations (2.11) are unitarily implementable iff the operators $K_{2}^{\alpha}$ and $K_{3}^{\alpha}$ are Hilbert-Schmidt. If this holds true there is a normed outgoing vacuum state $\Omega_{\text {out }}$ in the Fock space of the incoming fields $\phi^{\text {in }}$. The existence of $\Omega_{\text {out }}$ is an absolute necessity here because we want to define the fermion determinant as the scalar product $\left(\Omega_{\text {out }}, \Omega_{\text {in }}\right)$. Therefore the potential $A$ has to be selected in such a way that $K_{2}^{\alpha}$ and $K_{3}^{\alpha}$ are Hilbert-Schmidt. Using (2.13) we find that these conditions are equivalent to:

$$
\int_{-\infty}^{+\infty} d k|k| \operatorname{tr}\left(\tilde{V}_{\alpha}^{\dagger}(k) \tilde{V}_{\alpha}(k)\right)<\infty
$$

We work out this constraint for $\alpha=+$, remembering the expression (2.7) of $V_{+}$. Let $D=\left\{\mathbf{x} \mid x^{ \pm} \in[-L,+L]\right\}$; we observe that the values of $A_{-}$entering into (2.7) are given entirely in terms of $U$ if $\left|x^{+}\right|>L$. For $x^{+}<-L$ we find $V_{+}\left(x^{+}\right)=1$. If $x^{+}>L, V_{+}\left(x^{+}\right)$is determined by the discontinuity of $U$ across the cut $\Gamma$ :

$$
V_{+}\left(x^{+}\right)=\hat{U} \stackrel{\text { def }}{=} \lim _{\delta \triangleright 0} U^{\dagger}\left(\mathbf{w}^{\prime}\right) U\left(\mathbf{w}^{\prime \prime}\right) \quad \text { for } \quad x^{+}>L
$$

The points $\mathbf{w}^{\prime}$ and $\mathbf{w}^{\prime \prime}$ are on both sides of $\Gamma: w^{\prime+}=w^{\prime+}=x^{+}, w^{\prime 0}=-w^{\prime \prime}=\varepsilon>0$. It is readily seen that $\hat{U}$ is independent of $x^{+}$. These observations imply that the integral (2.12) extends effectively to $[-L, \infty)$ and that $\left(V_{+}(x)-\mathbb{1}\right)$ is constant for $x>L$. Consequently $\tilde{V}_{+}(k)$ has the singularity $(i / \sqrt{2 \pi})(1-\hat{U})(k-i \varepsilon)^{-1}$ at the origin and the integral (2.14) diverges at $k=0$ unless $\hat{U}=1$. In other words (2.14) forces $U$ to be single-valued in $D^{c}$ and $\left(V_{+}-1\right)$ to have a compact support. If 
moreover $\left(V_{+}-1\right) \in C_{0}^{1}\left(\mathbb{R}, \mathbb{C}^{N^{2}}\right)$, the asymptotic behaviour of $\tilde{V}_{+}$is such that the integral (2.14) converges.

The results obtained up to now are summarized in:

Proposition 2.1. If the field strength $F$ has compact support and if $A$ is such that $\left(V_{\alpha}-1\right)$ is in $C^{1}\left(\mathbb{R}, \mathbb{C}^{N^{2}}\right)$, the following three assertions are equivalent:

(i) the in-out automorphism (2.9) is unitarily implementable,

(ii) $\left(V_{\alpha}-1\right)$ have compact support,

(iii) the potential $A$ is globally a pure gauge outside a simply connected domain containing the support of $F$.

The last statement is equivalent to:

$$
P \exp \left(i g \oint_{C} d x^{\mu} A_{\mu}(\mathbf{x})\right)=\mathbb{1},
$$

where $C$ is a closed contour encircling $D$.

The condition (ii) and (iii) give a direct characterization of the potentials $A$ for which the in-out amplitude $\left(\Omega_{\text {out }}, \Omega_{\text {in }}\right)$ is defined.

In the abelian case, $G=U(1)$, condition (2.16) splits the admissible potentials into disjoint classes by quantizing the space-time flux of the electric field:

$$
\int d^{2} x F_{01}(\mathbf{x})=\frac{2 \pi n}{g}, \quad n \in \mathbb{Z} .
$$

In strong contrast to the abelian case we have those non-abelian groups $G$ which have a trivial first homotopy group. In that case the condition (iii) can be reformulated in a simple way. As $\pi_{1}(G)=0$, the map $U: D^{c} \rightarrow \rho(G)$ has $C^{1}$ extensions into the interior of $D$. Choosing one of them we get a $C^{1}$ map of the whole Minkowski space into $\rho(G)$; it defines a non-singular gauge transformation $A \rightarrow^{U^{\dagger}} A$ such that the new potential has its support in $D$. In the spirit of the terminology used in I, we call localizable a potential which has in its gauge orbit a potential with compact support, the latter is said to be localized.

Corollary 2.1. If the assumptions of Proposition 2.1 hold and if $\pi_{1}(G)=0$, the in-out automorphism (2.9) is unitarily implementable iff the potential is localizable.

In the abelian case, an admissible potential cannot be gauged away in $D^{c}$ if $n \neq 0$. A consequence of Corollary 2.1 is that the set of admissible potentials does not break up into disjoint pieces if $\pi_{1}(G)=0$.

In the following we restrict ourselves to the case $\pi_{1}(G)=0$ and ensure the existence of $\Omega_{\text {out }}$ by using a localized potential. Then $U=1$ and the fields $\phi^{\text {in }}$ and $\phi^{\text {out }}$ actually coincide with the asymptotic forms of $\psi$. Furthermore, the matrix function $R_{\alpha}$ are just the retarded solutions of (1.1).

Let $S$ be a unitary operator implementing (2.9):

$$
\phi^{\text {out }}(\mathbf{x})=S^{\dagger} \phi^{\text {in }}(\mathbf{x}) S, \quad \Omega_{\text {out }}=S^{\dagger} \Omega_{\text {in }} .
$$

The automorphism (2.9) defines $S$ up to an arbitrary phase. As long as we have no prescription for this phase, the phase of the fermion determinant, in particular 
its dependence on $A$, is undetermined. As is well known, it is the causality condition which removes this ambiguity (Yamagishi, ref. [5]). The machinery of the external field problem gives only information on the modulus of the determinant:

$$
|\operatorname{det} \not D[A]|=\left|\left(\Omega_{\text {out }}, \Omega_{\text {in }}\right)\right| \text {. }
$$

This is all we need for catching its zeros. A simple criterion established by Labonté [8] tells us whether $\Omega_{\text {out }}$ and $\Omega_{\text {in }}$ are orthogonal. In his terminology, $\left(\Omega_{\text {out }}, \Omega_{\text {in }}\right) \neq 0$ if the transformations (2.11) are weak, $\left(\Omega_{\text {out }}, \Omega_{\text {in }}\right)=0$ if at least one of them is strong. In a weak Bogoliubov transformation the kernels of $K_{1}^{\dagger}$ and $K_{4}^{\dagger}$ are both trivial, at least one of them is non-trivial in a strong transformation. The potentials producing a vanishing determinant are thus identified and one of our major goals is attained.

Proposition 2.2. Assume the potential $A$ to be localizable; the fermion determinant $\operatorname{det} D[A]$ vanishes iff at least one of the equations

$$
K_{1}^{\alpha^{\dagger}} f=0, \quad K_{4}^{\alpha^{\dagger}} g=0, \quad f, g \in L^{2}\left(\mathbb{R}_{+}, \mathbb{C}^{N}\right),
$$

has a non-trivial solution.

We explain briefly how (2.20) implies $\operatorname{det} D[A]=0$. Assume $g$ to be a solution of $K_{4}^{\alpha_{\dagger}^{\dagger}} g=0$. The second equation (2.11) gives $b_{\alpha}^{\text {out }}[g] \stackrel{\text { def }}{=}\left(b_{\alpha}^{\text {out }}[g]\right)^{\dagger}=a_{\alpha}^{\text {in }}\left[g^{*} K_{3}^{\alpha}\right]$. Consequently $b_{\alpha}^{\text {out }}[g] \Omega_{\text {in }}=0$ and, invoking the Fermi statistics, we see that there is a vector $\Psi$ such that $\Omega_{\text {in }}=b_{\alpha}^{\text {out }}[g] \Psi$. This shows that $\Omega_{\text {in }}$ and $\Omega_{\text {out }}$ are orthogonal.

The present situation is similar to the one encountered in the compactified euclidean case: there the zeros of the determinant correspond to zero modes of the Dirac operator. Here the zero mode equation is replaced by the integral equations (2.20). An advantage of the space-time approach is that there is a physical pair creation phenomenon which is responsible for the zeros of the determinant. To get a better insight into this mechanism we shall look more closely at the configuration of outgoing pairs the external potential creates in the ingoing vacuum. As a preparation we collect some results on the dimensions of various kernels.

Lemma 2.1. The integral operators $K_{1}^{\alpha}$ and $K_{4}^{\alpha}$ of a Bogoliubov transformation are such that:

$$
\begin{aligned}
& \operatorname{dim} \operatorname{ker} K_{1}^{\alpha}=\operatorname{dim} \operatorname{ker} K_{4}^{\alpha^{\dagger}}<\infty, \\
& \operatorname{dim} \operatorname{ker} K_{4}^{\alpha}=\operatorname{dim} \operatorname{ker} K_{1}^{\alpha^{\dagger}}<\infty .
\end{aligned}
$$

This is a direct consequence of the fact that the operator matrices

$$
K^{\alpha}=\left(\begin{array}{ll}
K_{1}^{\alpha} & K_{2}^{\alpha} \\
K_{3}^{\alpha} & K_{4}^{\alpha}
\end{array}\right)
$$

are unitary. In the special case at hand there are further relations among the dimensions of the kernels.

Lemma 2.2. If the integral operators $K_{n}^{\alpha}$ are given by (2.13) one has:

$$
N^{+}+N^{-}=\bar{N}^{+}+\bar{N}^{-}
$$


with $N^{\alpha}=\operatorname{dim} \operatorname{ker} K_{1}^{\alpha}, \bar{N}^{\alpha}=\operatorname{dim} \operatorname{ker} K_{4}^{\alpha}$. Furthermore, if $\pi_{1}(G)=0$ :

$$
N^{\alpha}=\bar{N}^{\alpha} \text {. }
$$

We are now ready to display the incoming vacuum in terms of outgoing entities. It is a coherent state of fermion-antifermion pairs which can be obtained following the techniques described in [9]. Due to the particular form of our $K$ 's we get a remarkably simple expression if $\pi_{1}(G)=0$ :

$$
\Omega_{\mathrm{in}}=e^{\imath \phi} \prod_{\alpha=+,-} \prod_{n}\left(\cos \theta_{n}^{\alpha}+\sin \theta_{n}^{\alpha} b_{\alpha}^{\text {out } \dagger}\left[g_{n}^{\alpha}\right] a_{\alpha}^{\text {out } \dagger}\left[f_{n}^{\alpha}\right]\right) \Omega_{\text {out }} .
$$

Here $\sin ^{2} \theta_{n}^{\alpha}$ are the simultaneous eigenvalues of the operators $K_{2}^{\alpha} K_{2}^{\alpha \dagger}$ and $K_{3}^{\alpha} K_{3}^{\alpha \dagger}$, $f_{n}^{\alpha *}$ and $g_{n}^{\alpha}$ are their normalized eigenfunctions, the phase $\phi$ is undetermined, as already noticed. The $f_{n}^{\alpha}$ s and $g_{n}^{\alpha}$ s forming two orthonormal sets, $\sin ^{2} \theta_{n}^{\alpha}$ is the inclusive probability for the external potential to create a fermion-antifermion pair $\left(f_{n}^{\alpha}, g_{n}^{\alpha}\right)$ in the incoming vacuum. Whenever one of the angles $\theta_{n}^{\alpha}$ is equal to $\pi / 2$ $(\bmod \pi)$ this probability is equal to 1 and $\Omega_{\text {in }}$ is orthogonal to $\Omega_{\text {out }}$, that is $\operatorname{det} \not D[A]=0$.

If one changes the external potential continuously, the angles $\theta_{n}^{\alpha}$ will move continuously too and it is plausible that any one of them can be brought to go through $\pi / 2$. This goes in the sense of our previous observation that the sets of potentials producing vanishing and non-vanishing determinants are not disconnected: we will come back to this point in Sects. 4 and 5. Appendix A contains an explicit example where the product over $n$ in (2.25) reduces to one factor.

By assumption, $K_{2}^{\alpha}$ and $K_{3}^{\alpha}$ are Hilbert-Schmidt operators; consequently $\left|\sin \theta_{n}^{\alpha}\right|$ is smaller than their Hilbert-Schmidt norm. Now, if the potential $A$ is small enough, the matrix functions $V_{\alpha}$ are everywhere close to $1, \tilde{V}_{\alpha}$ are small and $\left\|K_{n}^{\alpha}\right\|_{\text {H.S. }}<1$ $(n=2,3)$. Therefore, $\left|\sin \theta_{n}^{\alpha}\right|<1$ if the external potential is sufficiently small; zeros of the determinant show up for large potentials.

All potentials which produce the same pair of transition matrices $\left(V_{+}, V_{-}\right)$leave the same configuration of outgoing pairs after they are switched off. In particular, potentials such that $V_{+}=V_{-}=\mathbb{1}$ reabsorb all the pairs they create, their only effect being the phase $\phi$ between $\Omega_{\text {in }}$ and $\Omega_{\text {out }}$.

Without going into details, we describe briefly the structure of $\Omega_{\text {in }}$ in the abelian case $G=U(1)$. In this case $\Omega_{\text {in }}$ is orthogonal to $\Omega_{\text {out }}$ if the winding number $n$ defined in (2.17) is non-zero. This is due again to the presence, with probability 1 , of outgoing fermion-antifermion pairs, their number being now equal to $|n|$. Whereas in the non-abelian $\pi_{1}(G)=0$ case the outgoing particles were grouped into pairs whose partners were both right- or left-goers, we have now, if $n>0, n$ left-moving fermions and $n$ right-moving antifermions. This situation is reversed if $n<0$. The $|n|$ outgoing pairs carry the total outgoing chirality, equal to $2 n$, in conformity with the chiral anomaly. In contrast, the fact that the members of the pairs $\left(f_{n}^{\alpha}, g_{n}^{\alpha}\right)$ in the non-abelian vacuum (2.25) go in the same direction ensures here a vanishing outgoing $U(1)$ chirality, as it has to be. As the non-abelian chiral anomaly enters into a covariant continuity equation $\left(D^{\mu} j_{\mu}^{5}=-(g / 4 \pi) \varepsilon^{\mu \nu} F_{\mu \nu}\right)$, it has no simple implication on an outgoing non-singlet chiral charge which could be tested in (2.25). 


\section{Existence of the Causal Matrix Functions}

The properties of the fermion determinant obtained in the preceding section have been derived from the solution of the external field problem, without resorting to the formula for the sum of fermion loops established in I. At first sight, it is by no means obvious that the same properties result from this formula; that this is indeed the case will be established in the next sections. In the present section we answer the question of the existence of the ingredients our formula is made of.

We start with an outline of the basic results of $\mathrm{I}$. We assume $\pi_{1}(G)=0$, this implies that $\rho(G)$, our unitary representation of $G$, is a subgroup of $S U(N)$. Whereas our main tools in Sect. 2 were the retarded matrix functions $R_{\alpha}$ and their asymptotic forms, the transition matrices $V_{x}$, we have to work now with the causal matrix functions $T_{\alpha}$. They fulfil Feynman-Stueckelberg boundary conditions and, as a consequence, are not elements of $\rho(G)$. Because of that, they define the determinant as a complex quantity, fixing the phase which remained undetermined in Sect. 2.

Once the matrices $T_{\alpha}$ are known, the sum of fermion loops defines the following effective action:

$$
W[A] \stackrel{\text { def }}{=}-i \log \operatorname{det} D D[A]=I\left[T_{+}\right]+I\left[T_{-}^{-1}\right]+\left(g^{2} / 2 \pi\right) \int d^{2} x \operatorname{tr}\left(A_{+} A_{-}\right)(\mathbf{x}) .
$$

The functional $I$ is given by:

$$
\begin{aligned}
I[T] \stackrel{\text { def }}{=} & \frac{1}{4 \pi} \int d^{2} x \operatorname{tr}\left(\partial_{+} T \cdot T^{-1} \cdot \partial_{-} T \cdot T^{-1}\right)(\mathbf{x}) \\
& -\frac{1}{4 \pi} \int_{0}^{1} d t \int d^{2} x \operatorname{tr}\left(\partial_{t} T^{(t)} \cdot T^{(t)-1}\left[\partial_{+} T^{(t)} \cdot T^{(t)-1}, \partial_{-} T^{(t)} T^{(t)-1}\right]\right)(\mathbf{x}),
\end{aligned}
$$

the last integral has the structure of a Wess-Zumino term [10]; $T^{(t)}$ is a causal matrix function depending on a parameter $t$ and providing an interpolation between $T$ and the unit matrix: $T^{(1)}=T, T^{(0)}=1$.

As observed in the Introduction, our result is not strictly perturbative; if there are causal matrix functions for potentials for which the sum of loops diverges, (3.1-2) define a continuation of the effective action beyond the domain of validity of the perturbation expansion. One may ask if this continuation is correct. Furthermore, formula (3.1) was established in I in the case of a localized potential, but it makes sense also for a class of potentials extending to infinity; it is not certain that these are potentials for which the in-out automorphism of Sect. 2 is implementable.

The questions raised by the previous observations command a close comparison of the properties of the fermion determinant resulting from the external field problem and from the sum of loops. A crucial point will be to find out if formula (3.1) reproduces the zeros of the determinant identified in Sect. 2. This question is intimately related to the very existence of the causal matrix functions, a subject we address now. We shall find that the $T_{\alpha}$ 's exist whenever the potential $A$ produces a non-vanishing determinant according to Sect. 2.

To get a precise formulation of our problem we exploit the fact that the causal $T_{\alpha}$ and the retarded $R_{\alpha}$ obey the same differential equations (1.1); we can write:

$$
T_{\alpha}(\mathbf{x})=R_{\alpha}(\mathbf{x}) Q_{\alpha}\left(x^{\alpha}\right) .
$$


The matrix functions $R_{\alpha}$ exist for all integrable potentials and we have to find out if there are matrices $Q_{\alpha}$, each depending on a single light-cone variable, such that $T_{\alpha}$ are causal. Considering $Q_{+}$, the conditions it has to meet are obtained from the $x^{-} \rightarrow \mp \infty$ limits of (3.3). As:

$$
\lim _{x \rightarrow-\infty} R_{+}(\mathbf{x})=1, \quad \lim _{x^{-} \rightarrow+\infty} R_{+}(\mathbf{x})=V_{+}\left(x^{+}\right),
$$

we see that:

$$
\begin{aligned}
& Q_{+}\left(x^{+}\right)=\lim _{x^{-} \rightarrow-\infty} T_{+}(\mathbf{x}), \\
& \bar{Q}_{+}\left(x^{+}\right) \stackrel{\text { def }}{=}\left(V_{+} Q_{+}\right)\left(x^{+}\right)=\lim _{x \rightarrow+\infty} T_{+}(\mathbf{x}) .
\end{aligned}
$$

To get the asymptotic behaviour of $Q_{+}$we notice that:

$$
\lim _{x^{+} \rightarrow \pm \infty} R_{+}(x)=\lim _{x^{+} \rightarrow \pm \infty} V_{+}\left(x^{+}\right)=\mathbb{1} .
$$

Consistency with the perturbation expansion requires that $T_{+}(\mathbf{x})$ goes also to $\mathbb{1}$ for $x^{+} \rightarrow \pm \infty$. Consequently (3.3) and (3.6) give:

$$
\lim _{x^{+} \rightarrow \pm \infty} Q_{+}\left(x^{+}\right)=1 .
$$

Relations similar to (3.5) and (3.7) hold for $Q_{-}$. A direct consequence of (3.5) and the causality of $T_{\alpha}$ is that $Q_{\alpha}$ has only positive frequencies and $\bar{Q}_{\alpha}$ only negative ones. Our formulation needs to be slightly more cautious: (3.7) implies that the Fourier transforms of $Q_{\alpha}$ and $\bar{Q}_{\alpha}$ have a delta function singularity at the origin and what can be required unambiguously is that $\left(Q_{\alpha}-1\right)$, respectively $\left(\bar{Q}_{\alpha}-1\right)$ have frequencies of given signs.

We see that the external potential enters into the conditions determining $Q_{\alpha}$ only through the transition matrices $V_{\alpha}$; we call $Q_{\alpha}$ the causal complement of $V_{\alpha}$.

We may notice that if we rewrite (3.3) with $R_{\alpha}$ replaced by the advanced solution of (1.1) then $\bar{Q}_{\alpha}$ stands in place of $Q_{\alpha}$. This provides an alternative definition of $\bar{Q}_{\alpha}$.

In order to turn the conditions on $Q_{\alpha}$ into a well posed problem, we have to specify the assumed properties of $V_{\alpha}$ as well as the space to which $Q_{\alpha}$ has to belong. For this we don't need to have a potential with compact support; we relax this constraint and Eq. (2.7) gets replaced by:

$$
V_{+}\left(x^{+}\right)=P \exp \left[i g \int_{-\infty}^{+\infty} d y^{-} A_{-}\left(x^{+}, y^{-}\right)\right]
$$

with a similar expression for $V_{-}$. For the time being our only requirement will be that $\left(V_{\alpha}-1\right)$ have Fourier representations with integrable Fourier transforms. We define the following space of matrix functions having a finite limit at infinity:

$$
\begin{aligned}
\mathbf{M}= & \left\{M: \mathbb{R} \rightarrow \mathbb{C}^{N^{2}} \mid \lim _{x \rightarrow \pm \infty} M(x)=M_{\infty},\right. \\
& \left.\cdot M(x)=M_{\infty}+\int_{\mathbb{R}} d k \tilde{M}(k) e^{i k x}, \quad \tilde{M} \in L^{1}(\mathbb{R}, d k) \otimes \mathbb{C}^{N^{2}}\right\} .
\end{aligned}
$$

We choose a potential $A$ producing transition matrices $V_{\alpha}$ through (3.8) which are 
in $\mathbf{M}$ with $V_{\alpha, \infty}=1$; these $V_{\alpha}$ 's are uniformly continuous by virtue of the Riemann-Lebesgue lemma. As $V_{\alpha}(x)$ is an element of the unitary representation $\rho(G)$ of $G$, the $V_{\alpha}$ 's are in a subspace $\mathbf{V}$ of $\mathbf{M}$ :

$$
\mathbf{V}=\left\{V: \mathbb{R} \rightarrow \rho(G) \mid V \in \mathbf{M}, V_{\infty}=\mathbb{1}\right\} .
$$

We demand that the unknown causal complements $Q_{\alpha}$ belong to $\mathbf{M}$ (with $Q_{\alpha, \infty}=1$ ). This allows a precise formulation of the causality condition: the Fourier transform $\tilde{Q}_{\alpha}$ of $\left(Q_{\alpha}-\mathbb{1}\right)$ has to have their support on the positive axis $\mathbb{R}_{+}$. This defines a subspace $\mathbf{M}^{(+)}$of $\mathbf{M}$. If $\mathbb{R}_{+}$is replaced by $\mathbb{R}_{-}$, we obtain a complementary subspace $\mathbf{M}^{(-)}$which has to contain $\bar{Q}_{\alpha}$. We may now enunciate the problem of constructing the causal matrices $T_{\alpha}$. As we have separate problems for $T_{+}$and $T_{-}$, we can drop the index $\alpha$ for a while.

Problem 3.1. To find a pair of non-singular matrix functions $(Q, \bar{Q})$ such that:

(i) $Q \in \mathbf{M}^{(+)}, \widetilde{Q} \in \mathbf{M}^{(-)}, Q_{\infty}=\widetilde{Q}_{\infty}=\mathbb{1}$,

(ii) $\bar{Q}(x) Q^{-1}(x)=V(x), x \in \mathbb{R}$,

\section{$V$ being given, $V \in \mathbf{V}$.}

Once the hopefully unique solution of this problem is known, $T$ is given by (3.3).

The conditions (i) imply that the matrix functions $Q$ and $\bar{Q}$ are boundary values of analytic functions which are regular in the upper (lower) half-plane and go to 1 at infinity. Condition (ii) connects these functions through their boundary values and we have in fact a Riemann-Hilbert problem. This aspect of Problem 3.1 will be used explicitly only in the proof of the following lemma.

Lemma 3.1. If the pair $(Q, \bar{Q})$ is a solution of Problem 3.1, the matrices $Q(z), \bar{Q}(z)$ and $T(\mathbf{x})$ are elements of $\operatorname{Sl}(N, \mathbb{C})$.

Here $Q(z), \operatorname{Im} z \geqq 0(\bar{Q}(z), \operatorname{Im} z \leqq 0)$ designates the continuation of $Q(x)(\bar{Q}(x))$ into the upper (lower) half-plane.

Proof. To prove Lemma 3.1 we define:

$$
f(z)= \begin{cases}\operatorname{det} Q(z), & \operatorname{Im} z>0, \\ \operatorname{det} \bar{Q}(z), & \operatorname{Im} z<0 .\end{cases}
$$

This function is holomorphic in both half-planes and tends to 1 at infinity. It follows from condition (ii) and from $\operatorname{det} V(x)=1$ that $f$ is continuous across the real axis. It is therefore regular in the whole plane and has to be equal to 1 everywhere. That $T(x) \in S l(N, \mathbb{C})$ follows from (3.3).

What are the conditions under which Problem 3.1 has a solution? Remarkably, there is a "standard factorization theorem" due to Gohberg and Kreĭn (Theorem 7.3 in [11]) which provides the answer to that question. Adapted to our case, it gives:

Lemma 3.2. Let $M \in \mathbf{M}, M(x) \in \operatorname{Sl}(N, \mathbb{C})$ for $x \in \mathbb{R}$; this matrix function has a standard right factorization:

$$
M(x)=m^{(-)}(x) m(x) m^{(+)}(x)
$$

where $m^{( \pm)} \in \mathbf{M}^{( \pm)}$and $m^{( \pm)}(z) \in \operatorname{Sl}(N, \mathbb{C})$ for $\operatorname{Im} z \leqq 0$, respectively $\operatorname{Im} z \geqq 0 ; m$ is a 
diagonal unitary matrix:

$$
m_{j k}(x)=\left(\frac{x-i}{x+i}\right)^{\kappa_{j}} \delta_{j k} .
$$

The $\kappa_{k}$ 's are the uniquely defined right indices of $M$; they are integers, $\kappa_{1} \geqq$ $\kappa_{2} \geqq \cdots \geqq \kappa_{N}, \sum_{j} \kappa_{j}=-(1 / 2 \pi)[\arg \operatorname{det} M(x)]_{-\infty}^{\infty}$. This sum vanishes in our case because det $M(x)=1$.

Our assumptions on $V$ imply that this matrix function has a standard factorization (3.12): $V=v^{(+)} v v^{(-)}$.

Proposition 3.1. Problem 3.1 has a solution iff all the right indices of $V$ are zero. If this is so, the solution is unique.

Proof. $Q^{-1} \in \mathbf{M}^{(+)}$if $Q \in \mathbf{M}^{(+)}$as a consequence of Lemma 3.1. Therefore the left-hand side of condition (ii) has the form of a right standard factorization without middle term. If all indices of $V$ are zero, $v=1$ in its standard factorization and Problem 3.1 has the solution $Q(x)=\left(v^{(+)}(x)\right)^{-1}, \bar{Q}(x)=v^{(-)}(x)$. The boundary conditions $Q_{\infty}=\bar{Q}_{\infty}=1$ can be satisfied because $v^{( \pm)}$can be chosen such that $v_{\infty}^{(+)}=v_{\infty}^{(-)}=1$. The solution is unique because the right standard factorization of a matrix function with vanishing indices is unique once $v^{(+)}$is normalized by requiring $v_{\infty}^{(+)}=\mathbb{1}[11]$.

Suppose now that $v \neq \mathbb{1}$; this term can be absorbed into $v^{(+)}$and $v^{(-)}$in such a way that the new matrices are still in $\mathbf{M}^{(+)}$and $\mathbf{M}^{(-)}$. However, their analytic continuations have vanishing determinants at $z=+i$ and $z=-i$ and they cannot be identified with $(Q(z))^{-1}$ and $\bar{Q}(z)$ without contradicting Lemma 3.1 .

The example discussed in Appendix A provides an illustration of Proposition 3.1. The next Lemma tells us how to check whether a given element of $\mathbf{V}$ has only vanishing right indices.

Lemma 3.3. Let $V \in \mathbf{V}$, all its right indices vanish iff the homogeneous integral equation:

$$
(K \phi)(k) \stackrel{\text { def }}{=} \phi(k)+\int_{0}^{\infty} d p \tilde{V}(k-p) \phi(p)=0
$$

has no nontrivial solution in $L^{2}\left(\mathbb{R}_{+}, d k\right) \otimes \mathbb{C}^{N}$.

Here $\tilde{V}$ is the Fourier transform of $(V-1)$ as in (2.12).

The proof of this lemma can be extracted from [11], it is a direct consequence of the fact that the number of independent solutions of (3.14) is determined by the negative indices of $V$ :

$$
\operatorname{dim} \operatorname{ker} K=-\sum_{\substack{j \\ \kappa_{j}<0}} \kappa_{j} .
$$

As the sum of all the indices of $V$ is zero, there are negative indices as soon as they are not all zero and ker $K \neq 0$.

Remembering the expressions (2.13) of the kernels $K_{n}$ of the Bogoliubov transformations (2.11), we discover that $K=K_{4}$; the requirement that (3.14) has 
no solution is identical to:

$$
\text { ker } K_{4}=0 \text {. }
$$

Using Lemma 2.2 and Proposition 2.2 we see that this is precisely the condition ensuring a non-vanishing fermion determinant, as defined by the external field problem, when $\pi_{1}(G)=0$. A contact is thus established with Sect. 2 in a strikingly simple way: the causal matrix function $T$ exists whenever the fermion determinant is non zero.

The following proposition summarizes the results of this section.

Proposition 3.2. The causal matrix functions $T_{\alpha}$ exist and are unique iff the transition matrices $V_{\alpha}$ belong to a subspace $\mathbf{V}_{/ /}$of $\mathbf{V}$ :

$$
\mathbf{V}_{\|} \stackrel{\text { def }}{=}\{V \mid V \in \mathbf{V}, \text { ker } K=0\} \text {. }
$$

Taking up an observation already made in Sect. 2, we see that the causal matrices exist and $V_{\alpha} \in \mathbf{V}_{\|}$if the external potential is small. It is for large potentials that $T_{\alpha}$ may fail to exist. The membership of $V_{\alpha}$ to $\mathbf{V}_{/ /}$being an implicit constraint on $A$, it is not easy to tell whether a given large potential produces a non-vanishing determinant and has existing causal matrices.

If we go into the details we recognize that the present scheme is slightly broader than the one of Sect. 2. Even if we allow non-localized potentials in the external field problem, we have to maintain the implementability condition (2.14). Now there are elements in $\mathbf{V}_{/ /}$which do not fulfill this condition. However, we will have to introduce further restrictions on $V_{\alpha}$ in Sect. 4 and definitive conclusions would be premature.

\section{Topological Aspects and Interpolations}

Now that we know how to ensure the existence of the causal matrices $T_{\alpha}$ we can proceed to the evaluation of the effective action defined in (3.1). This requires the construction of the interpolations $T_{\alpha}^{(t)}$ appearing in the Wess-Zumino terms. This is by no means an easy task because we have no direct characterization of the set of all causal matrix functions. A straightforward procedure one could think of would be to start choosing an interpolating potential $A^{(t)}\left(t \in[0,1], A^{(0)}=0\right.$, $A^{(1)}=A$ ), then determine successively the corresponding retarded matrix functions $R_{\alpha}^{(t)}$, the transition matrices $V_{\alpha}^{(t)}$ and their causal complements $Q_{\alpha}^{(t)}$; finally $T_{\alpha}^{(t)}=R_{\alpha}^{(t)} Q_{\alpha}^{(t)}$ as in (3.3). The delicate point is the existence of $Q_{\alpha}^{(t)}$; according to Proposition 3.2 this requires $V_{\alpha}^{(t)}$ to be in $V_{/ /}$. In view of remarks made at the ends of Sect. 2 and 3, this will certainly be the case if $A^{(t)}$ is small enough and this can be so for all $t$ 's in $[0,1]$ if the given potential $A$ itself is small. Therefore, there is no obstacle in the construction of interpolating causal matrix functions $T_{\alpha}^{(t)}$ if the external potential is small. However, there is a problem if $A$ is large: it is not obvious how one has to choose $A^{(t)}$ in order to secure the existence of $T_{\alpha}^{(t)}$ for all $t$ 's in $[0,1]$. If we don't want to restrict the size of $A$, a more elaborate strategy is required. 
In order to find our way we notice that $V_{\alpha}^{(t)}$ defines a path in $V_{/ /}$. connecting the unit matrix to $V_{\alpha}\left(V_{\alpha}^{(0)}=1, V_{\alpha}^{(1)}=V_{\alpha}\right)$. For such a path to exist for every $V_{\alpha}$ in $V_{\| /}$, this space has to be path-connected. This rather trivial observation shows that we need properties of $\mathbf{V}_{\| /}$as a topological space and this fixes our first goal in this section. The results we shall collect are not just prerequisites for the construction of the interpolations, they also improve notably our comprehension of the Minkowski space fermion determinant.

We equip $\mathbf{M}$ with the norm:

$$
\|M\|=\sup _{\substack{1 \leqq ı . j \leqq N \\ x \in \mathbb{R}}}\left|M_{i j}(x)\right|,
$$

and we endow $\mathbf{V}_{/ /}$, which is a subset of $\mathbf{M}$, with the relative topology associated to this norm.

We call $\mathbf{V}_{\perp}$, the complement of $\mathbf{V}_{/ /}$in $\mathbf{V}$. If $V_{\alpha} \in \mathbf{V}_{\perp}, \alpha=+$ or (and),$- T_{\alpha}$ does not exist, and we expect the fermion determinant to vanish (this is certainly the case if (2.14) is fulfilled). Local properties of our spaces can be extracted from the work of Gohberg and Krein (Theorem 10.2 in [11]).

Proposition 4.1. (a) Let $V \in \mathbf{V}_{/ /}$, there is a neighborhood of $V$ in $\mathbf{V}$ which is entirely inside $\mathbf{V}_{/ /}$. (b) Let $V \in \mathbf{V}_{\perp}$, every neighborhood of $V$ in $\mathbf{V}$ contains elements belonging to $V_{\| /}$

Part (a) is a direct consequence of the quoted theorem, part (b) is a refinement of the result of Gohberg and Krein. Proposition 4.1 tells us that $\mathbf{V}_{/ /}$is dense in $\mathbf{V}$.

Remember that $V$ has only vanishing left indices if it is in $\mathbf{V}_{\| /}$, it has non-vanishing ones if it is in $\mathbf{V}_{\perp}$. Proposition 4.1 (a) expresses the stability of the situation in which all indices are zero. Part (b) tells us that a configuration with non-zero indices is unstable. This is already suggested by the expression (2.25) of the ingoing vacuum. If $K_{2}$ and $K_{3}$ are Hilbert-Schmidt, non-zero indices imply that some $\theta$ 's in $(2.25)$ are equal to $\pi / 2(\bmod \pi)$. A simple illustration of the instability of non-vanishing indices is given in Appendix A. The lesson of this is that the indices $\kappa_{j}$ do not label disconnected components of $\mathbf{V}$.

Now we turn to global aspects of $\mathbf{V}_{/ /}$. We shall get the properties we need only when the gauge group $G$ is $S U(N)$ and we shall arrive at them in an indirect way. First we shall demonstrate that the correspondence between a transition matrix $V$ and its causal complement $Q$ defines a homeomorphism $H$ between $\mathbf{V}_{/ /}$and the space $\mathbf{Q}^{(+)}$of causal complements:

$$
\mathbf{Q}^{( \pm)}=\left\{Q \mid Q \in \mathbf{M}^{( \pm)}, Q_{\infty}=\mathbb{1}, Q(x) \in \operatorname{Sl}(N, \mathbb{C}), x \in \mathbb{R}\right\} .
$$

Then we shall find that global properties of $\mathbf{Q}^{(+)}$are easily established; these properties are shared by $\mathbf{V}_{\|}$because of the homeomorphism $H$. Technically, the crucial result of this section is therefore:

Proposition 4.2. If $G=S U(N)$, the spaces $\mathbf{V}_{\| /}$and $\mathbf{Q}^{(+)}$are homeomorphic.

Proof. Our resolution of Problem 3.1 associates a unique $Q \in \mathbf{Q}^{(+)}$to every $V \in \mathbf{V}_{\| /}$ (such that $\bar{Q} \stackrel{\text { def }}{=} V Q \in \mathbf{Q}^{(-)}$). We write $Q=H(V)$ and have to show that the mapping $H$ is invertible. This is a consequence of: 
Lemma 4.1. If $G=S U(N)$ there is, for every matrix function $Q \in \mathbf{Q}^{(+)}$, a unique $\bar{Q} \in \mathbf{Q}^{(-)}$such that

$$
V \stackrel{\text { def }}{=} \bar{Q} Q^{-1}
$$

is in the space $\mathbf{V}_{/ /}$.

The proof of this lemma is given in Appendix B. The bijectivity of the mapping $H$ being established; it is straightforward to check its bicontinuity.

The reason why we were forced to take $G=S U(N)$ is that the range of $H$ is a subset of $\mathbf{Q}^{(+)}$, as defined in (4.2), if $G$ is a non-trivial subgroup of $S U(N)$, and we have no direct characterization of this subset. We assume $G=S U(N)$ from now on.

Our information on the global structure of our spaces is contained in the following proposition:

Proposition 4.3. The topological space $\mathbf{Q}^{(+)}$is contractible to the unit matrix function; the same is true for $\mathbf{V}_{/ /}$.

Proof. We have to show that the identity map of $\mathbf{Q}^{(+)}$is homotopic to the constant $\operatorname{map} \mathbf{Q}^{(+)} \rightarrow \mathbb{1}$. Our proof exploits the analyticity properties of the elements of $\mathbf{Q}^{(+)}$. If $Q \in \mathbf{Q}^{(+)}$it is readily seen that the restriction of its analytic continuation to the horizontal line $\operatorname{Im} z=\alpha>0$ defines a matrix function $Q_{\alpha}$ belonging also to $\mathbf{Q}^{(+)}$, $Q_{\alpha}(x)=Q(x+i \alpha), x \in \mathbb{R}$. Consequently the continuous map $\Phi:[0,1] \times \mathbf{Q}^{(+)} \rightarrow \mathbf{Q}^{(+)}$ defined by:

$$
\Phi(t, Q)(x) \stackrel{\text { def }}{=} Q(x+i((1-t) / t)), \quad x \in \mathbb{R}, \quad t \in[0,1]
$$

provides the required homotopy:

$$
\Phi(t=1, Q)=Q, \quad \Phi(t=0, Q)=\mathbb{1} .
$$

That $\mathbf{V}_{\|}$is contractible too follows from the fact that $\mathbf{V}_{\|}$and $\mathbf{Q}^{(+)}$are homeomorphic.

The very reason why localized potentials have been abandoned is similar to the reason which forced the restriction to $G=S U(N)$. If $A$ is localized, $\left(V_{\alpha}-\mathbb{1}\right)$ have compact supports and this property defines a subset of $\mathbf{V}_{\|}$. The homeomorphism $H$ maps it onto a subset of $\mathbf{Q}^{(+)}$. As we have no direct characterization of this last subset, we cannot prove it is contractible. This means that if we have a large localized potential $A$ we are not sure that there is a localized interpolating potential $A^{(t)}$ such that $T_{\alpha}^{(t)}$ exist for all $t$ in $[0,1]$. If we consider $A_{-}$, the preceding remarks apply only to its $x^{+}$-dependence. We may well ask $A_{\text {- to }}$ to have compact support with respect to $x^{-}$, contained in $[-L,+L]$, and construct an interpolation with the same property. This is what we shall do.

After these topological preparations, we may address the interpolation problem, that of $T_{+}$to be specific. Dropping indices, the given external potential $A$ defines the retarded matrix function $R$, the transition matrix function $V$, which we assume to belong to $\mathbf{V}_{/ /}$, and its causal complement $Q, Q \in \mathbf{Q}^{(+)}$. Compared to the scheme 
outlined at the beginning of this section, the procedure we work out now goes backwards, starting rather than ending with $Q^{(t)}$.

Our construction breaks up into four steps:

(i) The interpolating causal complement $Q^{(t)}\left(Q^{(0)}=1, Q^{(1)}=Q\right)$ is determined by means of the homotopy $\Phi$ defined in (4.5):

$$
Q^{(t)}=\Phi(t, Q), \quad Q=H(V)
$$

(ii) The interpolating transition matrix $V^{(t)}$ is obtained from $Q^{(t)}$ through the inverse of the map $H: \mathbf{V}_{/ /} \rightarrow \mathbf{Q}^{(+)}$:

$$
V^{(t)}=H^{-1}\left(Q^{(t)}\right)
$$

(iii) Construction of an interpolating retarded matrix $R^{(t)}\left(x^{+}, x^{-}\right)$fulfilling the following boundary conditions:

$$
\begin{aligned}
& R^{(0)}\left(x^{+}, x^{-}\right)=R^{(t)}\left( \pm \infty, x^{-}\right)=R^{(t)}\left(x^{+},-L\right)=\mathbb{1}, \\
& R^{(1)}\left(x^{+}, x^{-}\right)=R\left(x^{+}, x^{-}\right), R^{(t)}\left(x^{+},+L\right)=V^{(t)}\left(x^{+}\right) .
\end{aligned}
$$

(iv) The interpolating causal matrix $T^{(t)}$ is given by:

$$
T^{(t)}\left(x^{+}, x^{-}\right)=R^{(t)}\left(x^{+}, x^{-}\right) Q^{(t)}\left(x^{+}\right) .
$$

Although it is not necessary to know the interpolating potential, it can be computed from $A_{-}^{(t)}=-(i / g) \partial_{-} R^{(t)} \cdot R^{(t)-1}$.

If this construction goes through, the effective action is obtained by inserting $T_{+}^{(t)}$, respectively $T_{-}^{(t)-1}$ into the right-hand side of (3.2). At this point we can no longer ignore that $I[T]$ contains not only the interpolation $T^{(t)}$ but also its first order derivatives with respect to $x^{+}, x^{-}$and $t$. We didn't care about differentiability properties until now, but the effective action may fail to exist if such properties are not ensured. This means that we shall have to replace $\mathbf{V}_{/ /}$and $\mathbf{Q}^{(+)}$by suitable subspaces of differentiable matrix functions. As we were mainly concerned so far with $V$ and $Q$, there is another gap we left open and which has to be filled now: we didn't specify the space to which the retarded matrix $R$ has to belong.

Having again the case $\alpha=+$ in mind, the three spaces we shall need are defined as follows:

$$
\begin{aligned}
\hat{\mathbf{Q}}^{(+)}= & \left\{Q \mid Q \in \mathbf{Q}^{(+)}, \tilde{Q} \in L^{\infty}\left(\mathbb{R}_{+}, d k\right) \otimes \mathbb{C}^{N^{2}}, k \widetilde{Q} \in L^{1}(\mathbb{R}+, d k) \otimes \mathbb{C}^{N^{2}}\right\}, \\
\hat{\mathbf{V}}= & \left\{V \mid V \in \mathbf{V}_{\| /}, \tilde{V} \in L^{\infty}(\mathbb{R}, d k) \otimes \mathbb{C}^{N^{2}}, k \tilde{V} \in L^{1}(\mathbb{R}, d k) \otimes \mathbb{C}^{N^{2}}\right\}, \\
\hat{\mathbf{V}}_{/ /}= & \left\{V \mid V \in \hat{\mathbf{V}} \cap \mathbf{V}_{\| /}\right\}, \\
\hat{\mathbf{R}}= & \{R: \mathbb{R} \times[-L,+L] \\
& \left.\rightarrow S U(N) \mid \widetilde{R}\left(\cdot, x^{-}\right) \in \hat{\mathbf{V}}, \partial_{-} \widetilde{R}\left(\cdot, x^{-}\right) \in L^{\infty}(\mathbb{R}, d k) \otimes \mathbb{C}^{N^{2}}\right\} .
\end{aligned}
$$

Similarly as in (3.9), $\left(Q\left(x^{+}\right)-1\right),\left(V\left(x^{+}\right)-1\right)$ and $\left(R\left(x^{+}, x^{-}\right)-1\right)$ are the Fourier transforms of $\widetilde{Q}(k), \widetilde{V}(k)$ and $\widetilde{R}\left(k, x^{-}\right)$with respect to $k$. The following lemma completes Proposition 4.2 and asserts that whenever $V$ is in $\hat{\mathbf{V}}_{/ /}$its causal complement $Q$ is in $\hat{\mathbf{Q}}^{(+)}$and vice versa. 
Lemma 4.2. The map $H$ defines a homeomorphism between $\hat{\mathbf{V}}_{/ /}$and $\hat{\mathbf{Q}}^{(+)}$.

Conditions for the existence of the effective action $W$ are given in the next proposition:

Proposition 4.4. Assume $A_{-}$is such that $R \in \hat{\mathbf{R}}$ and $V \in \hat{\mathbf{V}}_{/ /}$. Then there are interpolating matrix functions $Q^{(t)} \in \hat{\mathbf{Q}}^{(+)}, V^{(t)} \in \hat{\mathbf{V}}_{\| /}, R^{(t)} \in \hat{\mathbf{R}}$ with $\partial_{t} \widetilde{R}^{(t)}, \partial_{t} \widetilde{V}^{(t)}, \partial_{t} \widetilde{Q}^{(t)} \in$ $L^{1}(\mathbb{R}, d k) \otimes \mathbb{C}^{N^{2}}$ and the functional $I[T]$ defined in (3.2) exists.

We comment on this proposition without displaying its proof.

1. The interpolations are constructed according to the four step procedure outlined before. $Q^{(t)}$ given by (4.4) is generally not differentiable at $t=0$ and has to be regularized there (by means of a partition of unity).

2. The existence of $R^{(t)}$ is essentially a consequence of the fact that the second homotopy group of $S U(N)$ is trivial. $R^{(t)}$ defines a map of a 3-dimensional domain $\Delta$ into $S U(N)$ with given boundary values (4.8):

$$
\Delta=\left\{t \in[0,1], x^{+} \in \mathbb{R}, x^{-\cdots} \in[-L,+L]\right\}
$$

These specify a continuous map $\partial \Delta \rightarrow S U(N)$ which has extensions to the interior of $\partial \Delta$ because $\pi_{2}(S U(N))=0$. The set of these extensions contains differentiable ones.

3. In the definitions (4.10), the conditions on $k \widetilde{Q}, k \widetilde{V}, k \widetilde{R}$ and $\partial_{-} \tilde{R}$ secure the existence of first order derivatives with respect to $x^{+}$and $x^{-}$with suitable integrability properties.

4. If $R \in \hat{\mathbf{R}}, R\left(\cdot, x^{-}\right)$is not necessarily in $\mathbf{V}_{/ /}$; it may belong to $\mathbf{V}_{\perp}$ for some values of $x^{-}$in $(-L,+L)$. It is only its end-point value $R(\cdot, L)=V$ which is required to belong to $\hat{\mathbf{V}}_{/ /}$in Proposition 4.4. This is related to the fact that the causal complement $Q$ is determined by $V$ and does not depend on the details of $R\left(\cdot, x^{-}\right)$as a function of $x^{-}$.

5. The interpolations whose existence is ascertained in Proposition 4.4 are not unique. The value of $W[A]$ does not change under small modifications of the interpolations; this was shown in $\mathrm{I}$. However, as $\pi_{3}(S U(N))=\mathbb{Z}$, there may be non-homotopic interpolations and the corresponding effective actions would differ by multiples of $2 \pi$.

6. In view of a functional integral over the gauge potential $A$ we are interested in obtaining an effective action under conditions on $A$ which are as weak as possible. The final restrictions on $A$ we arrive at are contained in Proposition 4.4. These are rather implicit constraints on $A$ and they are not optimal in the sense that membership of the spaces (4.10) is a sufficient condition for the existence of the effective action but is not necessary. However, our experience tells us that there is not much room left once one agrees to start with the spaces $\mathbf{M}^{( \pm)}$which are natural choices in the context of Problem 3.1.

An observation related to the last comment is that the transition matrix $V$ fulfills the implementability condition (2.14) if it is produced by a retarded matrix belonging to $\hat{\mathbf{R}}$. The requirement of differentiability removes the discrepancy noticed at the end of Sect. 3; it is worthwhile to keep this in a lemma.

Lemma 4.3. The in-out automorphism of the external field problem is unitarily implementable whenever the transition matrix $V$ is in $\hat{\mathbf{V}}$. 
We see now that within our assumptions the formula (3.1) for the effective action makes sense whenever one has a non-vanishing fermion determinant, as defined by the external field problem. This does not yet prove that our formula is correct: the example in Appendix A provides a first indication that this is likely to be the case. In this example we may vary $V$ in $\mathbf{V}_{\|}$and let it go to an element of $\mathbf{V}_{\perp}$; whereas $V$ behaves smoothly in this process, its causal complement $Q$ explodes with the effect that $\operatorname{Im} W$ diverges and det $D$ tends to zero, as it should. Further evidence of the correctness of formula (3.1) will be presented in the next section.

\section{Further Results and Concluding Remarks}

If a potential produces a vanishing fermion determinant according to the external field problem this potential is also a singular point of the effective action provided by the sum of fermion loops. Up to now this is the major outcome of the confrontation of our two approaches to the fermion determinant. This confrontation can be pushed further. In addition to its zeros, the external field problem gives also the modulus of the determinant and one may check if the effective action gives the same result. Even if one has no serious doubt about this identity, its proof is a challenging exercise because one starts with two very disparate expressions. We explain briefly how to proceed.

Formula (2.25) gives

$$
|\operatorname{det} D[A]|=\prod_{\alpha= \pm} \prod_{n}\left|\cos \theta_{n}^{\alpha}\right| \text {. }
$$

We discuss the positive chirality factor and drop indices. In view of the expression (3.1) of $W[A]$ we have to establish the following equality:

$$
\operatorname{Im} I[T]=-\frac{1}{2} \operatorname{Tr} \log K_{4} K_{4}^{\dagger} .
$$

We have used the fact that the set $\left\{\cos ^{2} \theta_{n}\right\}$ is the spectrum of $K_{4} K_{4}^{\dagger}$.

The interpolating potential $A^{(t)}$ obtained in Sect. 4 defines an interpolating operator $K_{4}^{(t)}$ connecting the identity $\left(=K_{4}^{(0)}\right)$ to $K_{4}\left(=K_{4}^{(1)}\right)$. This allows a more tractable form of the right-hand side of (5.2):

$$
\operatorname{Im} I[T]=-\frac{1}{2} \int_{0}^{1} d t \operatorname{Tr}\left(K_{4}^{-1} \partial_{t} K_{4}+\operatorname{adj}\right)
$$

Here $K_{4}$ stands for $K_{4}^{(t)}$. The functional $I[T]$ is given in terms of the $x$-space causal matrix function $T$ whereas the operator $K_{4}$ defined in $(2.13)$ acts on $L^{2}\left(\mathbb{R}_{+}, d k\right)$. To verify (5.3) we shall write its right-hand side in $x$-space by means of a Fourier transformation. To this end we must extend $K_{4}$ to an operator acting on functions defined on the whole real $k$-axis. Instead of (2.13) we write:

$$
K_{4}\left(k, k^{\prime}\right)=\delta\left(k-k^{\prime}\right)+\theta(k) \tilde{V}\left(k-k^{\prime}\right) \theta\left(k^{\prime}\right) .
$$

This substitution does not change the value of the right-hand side of (5.3). Switching to $x$-space, (5.4) gives:

$$
K_{4}\left(x, x^{\prime}\right)=\left(P^{(-)}+P^{(+)} V P^{(+)}\right)\left(x, x^{\prime}\right) .
$$


Here $P^{(+)}$are the projectors onto positive, respectively negative frequencies:

$$
P^{( \pm)}\left(x, x^{\prime}\right)= \pm \frac{i}{2 \pi} \frac{1}{x-x^{\prime} \pm i \varepsilon}
$$

and the transition matrix $V$ is meant as a multiplication operator: $(V \phi)(x)=$ $V(x) \phi(x)$.

Besides $K_{4}$ we need also its inverse; it is at this point that the contact between the external field problem and the sum of loops is established in a rather unexpected way. It is the knowledge of the causal complement $Q$ of the transition matrix $V$ which provides the expression of $K_{4}^{-1}$ we need. It is easy to check that this operator is given by:

$$
K_{4}^{-1}=P^{(-)}+Q P^{(+)} \bar{Q}^{-1}
$$

(remember that $\bar{Q}=V Q$ ).

Inserting (5.5) and (5.7) into (5.3) we get:

$$
\operatorname{Im} I\left[T_{+}\right]=\frac{1}{2 \pi} \operatorname{Im} \int_{0}^{1} d t \int_{-\infty}^{+\infty} d x^{+} \operatorname{tr}\left(\partial_{+} Q_{+}^{(t)} Q_{+}^{(t)-1} V_{+}^{(t)} \partial_{t} V_{+}^{(t)}\right)\left(x^{+}\right) .
$$

To verify this relation, we have to evaluate its left-hand side with $I\left[T_{+}\right]$given by (3.2). Replacing $T_{+}^{(t)}$ by $R_{+}^{(t)} Q_{+}^{(t)}$ one finds that the integrand of the imaginary part of the Wess-Zumino term is a sum of total derivatives with respect to $x^{+}, x^{-}$and $t$. This leads to

$$
\begin{aligned}
\operatorname{Im} I\left[T_{+}\right]= & \frac{1}{4 \pi} \operatorname{Im} \int_{0}^{1} d t \int_{-\infty}^{+\infty} d x^{+} \operatorname{tr}\left[\partial_{+} Q_{+}^{(t)} Q_{+}^{(t)-1} V_{+}^{(t) \dagger} \partial_{t} V_{+}^{(t)}\right. \\
& \left.-\partial_{t} Q_{+}^{(t)} Q_{+}^{(t)-1} V_{+}^{(t) \dagger} \partial_{+} V_{+}^{(t)}\right]\left(x^{+}\right) .
\end{aligned}
$$

The structure of this expression is already very close to that of (5.8). Using the analyticity properties of $Q_{+}^{(t)}$ and $\bar{Q}_{+}^{(t)}$ one finds that the second term of the integrand in the right-hand side of (5.9) gives the same contribution to $\operatorname{Im} I\left[T_{+}\right]$as the first term; this proves that $(5.8)$ is correct.

The identity of our two expressions of $|\operatorname{det} \not D|$ comes out in a remarkable and intriguing way. The projectors $P^{( \pm)}$in (5.5) have their origin in the strict correlation of the chirality and the sign of the momentum of two-dimensional massless fermions. Equation (5.5) implies that $K_{4}^{-1}$ too has the structure $\left(P^{(-)}+P^{(+)} X P^{(+)}\right)$. The right-hand side of (5.7) has indeed this structure: $Q P^{(+)} \bar{Q}^{-1}=P^{(+)} Q P^{(+)} \bar{Q}^{-1} P^{(+)}$ because $Q$ and $\bar{Q}^{-1}$ are regular in the upper, respectively lower half-plane. These last properties are consequences of causality, which has manifestly nothing to do with a chirality-momentum correlation. The causal complements $Q$ are responsible for the imaginary part of $W(\operatorname{Im} W=0$ if $Q=0)$ and their specific causal properties have the effect that $\operatorname{Im} W$ has the value predicted by the solution of the external field problem, a solution obtained without invoking causality in an explicit way.

The last result provides also an a posteriori justification of our definition of the in-out correspondence in Sect. 2. The outcome of this section depends crucially on the identification of $\Omega_{\text {in }}$ and $\Omega_{\text {out }}$ with the vacua of the gauge invariant free fields $\phi^{\text {in }}$ and $\phi^{\text {out }}$. Although this choice is quite natural, it is comforting to have it confirmed by the sum of loops. 
Once one knows that the fermion determinant has zeros one becomes curious about their distribution. As we have learned, the fact that det $D[A]$ vanishes depends only on properties of the transition matrices $V_{\alpha}$ and there is a whole class of potentials producing the same transition matrices. These classes are subsets of the space $\mathbf{A}_{\perp}$ referred to in the Introduction. As they are difficult to handle, it is advisable to localize the zeros in the space of transition matrices. If $\operatorname{det} D[A]=0$ the causal matrices do not exist and $V_{\alpha} \in \mathbf{V}_{\perp}(\alpha=+$ or (and) -$)$. Proposition 4.1 gives a first information on the way $\mathbf{V}_{\perp}$ is embedded in $\mathbf{V}$. The results we present now provide a deeper insight into the structure of $\mathbf{V}_{\perp}$ and $\mathbf{V}_{\| /}$. Let $\boldsymbol{\kappa}$ designate the collection of decreasing indices $\left\{\kappa_{j}\right\}_{j=1}^{N}$ appearing in the right standard factorization (3.12) of a transition matrix $V$. The manifold $\mathbf{V}$ is the union of the submanifolds $\mathbf{V}_{\boldsymbol{\kappa}}$ containing all matrices with a given $\boldsymbol{\kappa}$ : clearly: $\mathbf{V}_{/ /}=\mathbf{V}_{\mathbf{0}}$ and:

$$
\mathbf{V}_{\perp}=\bigcup_{n \neq 0} \mathbf{V}_{\boldsymbol{\kappa}} .
$$

Using results in [11], it can be shown that the complex codimension of $\mathbf{V}_{\boldsymbol{\kappa}}$ is finite, non-vanishing if $\boldsymbol{\kappa} \neq \mathbf{0}$, equal to:

$$
d(\boldsymbol{\kappa})=\sum_{k>j}\left|\kappa_{J}-\kappa_{k}\right|-v(\boldsymbol{\kappa})
$$

where $v(\boldsymbol{\kappa})$ is the number of pairs $\left(\kappa_{j}, \kappa_{k}\right), j<k$ such that $\kappa_{j}>\kappa_{k}$. In particular $d(\mathbf{0})=\mathbf{0}, \mathbf{V}_{\mathbf{0}}$ is dense in $\mathbf{V}$ as already stated in Proposition 4.1.

Each $\mathbf{V}_{\boldsymbol{K}}$ is an infinite dimensional stratum of a stratification of $\mathbf{V}$. To specify this structure we have to introduce a partial ordering of the $\boldsymbol{\kappa}$ 's. According to [11] we define an elementary operation acting on the $\boldsymbol{\kappa}$ 's. If for a given $\boldsymbol{\kappa}$, there are two integers $j$ and $k(1 \leqq j<k \leqq N)$ such that

$$
\kappa_{j}^{\prime}=\kappa_{j}-1, \quad \kappa_{k}^{\prime}=\kappa_{k}+1, \quad \kappa_{l}^{\prime}=\kappa_{l} \quad \text { for } \quad l \neq j, \quad l \neq k,
$$

form a new decreasing sequence $\boldsymbol{\kappa}^{\prime}, \boldsymbol{\kappa}^{\prime}$ is said to be obtained from $\boldsymbol{\kappa}$ by an elementary operation. If $\boldsymbol{\kappa}^{\prime}$ is obtained from $\boldsymbol{\kappa}$ by a sequence of elementary operations, one writes $\boldsymbol{\kappa}>\boldsymbol{\kappa}^{\prime}$ and this defines the required partial ordering. The fact that $\mathbf{V}$ is stratified by the $\mathbf{V}_{\boldsymbol{\kappa}}$ 's means that every $\mathbf{V}_{\boldsymbol{\kappa}^{\prime}}$ with $\boldsymbol{\kappa}^{\prime} \geqq \boldsymbol{\kappa}$ is contained in the closure of $\mathbf{V}_{\boldsymbol{\kappa}}: \mathbf{V}_{\boldsymbol{\kappa}^{\prime}} \subset \overline{\mathbf{V}}_{\boldsymbol{\kappa}}$. Furthermore, every $V$ belonging to $\mathbf{V}_{\boldsymbol{\kappa}^{\prime}}$ has for each $\boldsymbol{\kappa} \leqq \boldsymbol{\kappa}^{\prime}$ a neighborhood in $\mathbf{V}_{\boldsymbol{\kappa}^{\prime}}$ which is entirely in $\mathbf{V}_{\boldsymbol{\kappa}}$. This local result is again a refinement of Proposition 4.1. All this shows that $\mathbf{V}_{\perp}$ has a very rich structure. A similar stratification appears in the study of the wave functionals of the Wess-Zumino-Witten models [12].

We may conclude by saying that the properties of the non-abelian two dimensional Minkowski space fermion determinant we have established, particularly our complete characterization of its zeros, improve substantially our understanding of this object. We list a few salient features.

1. In our two approaches we find that $\operatorname{det} D[A]$ is defined only for a restricted class of admissible external potentials. These are the potentials for which the in-out automorphism of the external field problem is unitarily implementable.

2. It is crucial that the building blocks of the effective action are causal in the 
sense of Feynman and Stueckelberg. If instead of $T_{ \pm}$we would use $R_{ \pm}$in (3.1) the effective action would be real, ignoring the pairs created by the external potential. It is the use of the causal matrix functions which leads to the physically correct complex effective action with singularities located at the zeros of the determinant.

3. The causal complements $Q$ are uniquely determined by the transition matrices $V$ which express global integral characteristics of the gauge potential rather than the details of its local behaviour. There are large families of potentials sharing the same transition matrices and causal complements and for which the effective action has a fixed imaginary part. The real part of the effective action, that is the phase of the determinant distinguishes the potentials belonging to a given family.

4. The distribution of the zeros exhibits an intricate structure which is possible because these zeros do not split into distinct disconnected classes. In terms of transition matrices, the zeros are imbedded in the space of all these matrices forming submanifolds of finite non-vanishing codimension labeled by sets of integer indices. Our understanding of the distribution of the zeros is still incomplete and we do not know what role they play in a functional integration over the gauge potential.

5. Although there is no direct connection between the minkowskian and euclidean external field problems, one may look for possible similarities. To this end we notice that when one is dealing with the transition matrices $V_{\alpha}\left(x^{\alpha}\right)$, the real $x^{\alpha}$-axis can be mapped onto $S^{1}$. This is so because $V_{\alpha}\left(x^{\alpha}\right)$ go to the same value 1 for $x^{\alpha} \rightarrow+\infty$ and $x^{\alpha} \rightarrow-\infty$. This means that $V_{\alpha}$ are elements of a loop group [13], i.e. mappings from $S^{1}$ into the gauge group. The indices $\kappa_{j}$ classify the elements of this group. In the euclidean problem, compactified on $S^{2}$, transition functions connecting quantities defined on the upper and lower hemispheres play a crucial role [4]. They are primarily defined on the equator and are also elements of a loop group. Consequently, the manifold of euclidean zero modes and the manifold of zeros of the Minkowski space determinant have equivalent stratifications.

\section{Appendix A. An Explicit Example}

We present an example where all our constructions can be done explicitly. It is obtained by considering transition matrices whose elements are rational functions and choosing the simplest case: $G=S U(2)$ and a matrix with two poles which we set at $x= \pm i$. Each of these matrices has the form $U V(x) U^{\dagger}$, where $U$ is a constant matrix and $V(x)$ is given by:

$$
V(x)=\left(\begin{array}{cc}
\frac{x+i \cos \omega}{x+i} & \frac{e^{i v} \sin \omega}{x-i} \\
-\frac{e^{-i v} \sin \omega}{x+i} & \frac{x-i \cos \omega}{x-i}
\end{array}\right)
$$

where $\omega \in[0, \pi], v \in[0,2 \pi)$. Without loss of generality we restrict ourselves to this 
particular matrix function. Our first goal is the explicit determination of the form (2.25) of the ingoing vacuum. The spectrum of the operator $K_{3} K_{3}^{\dagger}$ is $\left\{0, \sin ^{2}(\omega / 2)\right\} ; 0$ is an infinitely degenerate eigenvalue and $\sin ^{2}(\omega / 2)$ is nondegenerate. In $x$-space its eigenvector is:

$$
\phi(x)=(1 / \pi) \frac{1}{x+i}\left(\begin{array}{c}
i e^{i v} \sin (\omega / 2) \\
\cos (\omega / 2)
\end{array}\right) .
$$

There is a second eigenvector of $K_{3} K_{3}^{\dagger}$ proportional to $(x+i)^{-1}$ with eigenvalue 0 . The remaining eigenvectors corresponding to this last eigenvalue span the space of all vectors whose elements are regular in $\operatorname{Im} x>0$ and have a zero at $x=i$. If we identify (A.1) with the chirality $\alpha$ transition matrix, the corresponding product in the right-hand side of (2.25) reduces to a single non-trivial factor with $\theta=\omega / 2$, $f, g \sim \exp (-k)$. In particular $|\operatorname{det} \not D[A]|=\left|\left(\Omega_{\text {out }}, \Omega_{\text {in }}\right)\right|=\cos (\omega / 2)$; the determinant is a continuous function of $\omega$ which vanishes at $\omega=\pi$.

We turn now to the right standard factorization of (A.1). If $\omega \neq \pi, V$ has vanishing indices $\kappa_{1}$ and $\kappa_{2}$, belongs to $\mathbf{V}_{/ /}$and:

where

$$
V(x)=\bar{Q}(x) Q^{-1}(x)
$$

$$
\begin{aligned}
& Q(x)=\mathbb{1}+2 e^{-i v} \operatorname{tg}(\omega / 2)\left(\begin{array}{ll}
0 & 0 \\
1 & 0
\end{array}\right) \frac{1}{x+i^{5}} \\
& \bar{Q}(x)=\mathbb{1}-2 i \sin ^{2}(\omega / 2)\left(\begin{array}{cc}
1 & i e^{\imath v} \operatorname{ctg}(\omega / 2) \\
i e^{-i v} \operatorname{tg}(\omega / 2) & -1
\end{array}\right) \frac{1}{x-i} .
\end{aligned}
$$

For the particular value $\omega=\pi$ the matrix $V$ is diagonal, $v_{+}=v_{-}=\mathbb{1}$, its indices are non-zero, $\boldsymbol{\kappa}=(1,-1)$ and $V$ is in $\mathbf{V}_{\perp}$. This illustrates the instability of a configuration of non-zero indices. If we look at the form of (A.1) and the domain of variation of its parameters $\omega$ and $v$, we see that every $V$ is specified by a point $P$ of the two-sphere of $S^{2}: \boldsymbol{\kappa}=\mathbf{0}$ on the whole sphere except at the south pole. Furthermore, we observe that $Q$ and $\bar{Q}$ have non-diagonal matrix elements which diverge if $P$ moves to the south pole. One checks that this produces the logarithmic divergence of $\operatorname{Im} W$ as $\omega \rightarrow \pi$ which forces $\operatorname{det} \not D$ to vanish at $\omega=\pi$.

If we want to compute the real part of $W$ we have to fix the external potential and not only the asymptotic forms $V_{ \pm}$of $R_{ \pm}$. Considering again the positive chirality components, we fix $A_{-}$by choosing a retarded matrix function $R_{+}$given by the right-hand side of (A.1) with $\omega$ and $v$ replaced by suitable functions of $x^{-}$. The $t$-dependent interpolations are obtained by introducing a $t$-dependence into $\omega\left(x^{-}\right)$and $v\left(x^{-}\right)$. One verifies that the functional $I\left[T_{+}\right]$(3.2) does not depend on the interpolation one chooses up to a known possible additive ambiguity of a multiple of $2 \pi$ [1]. This multivaluedness is discussed in detail elsewhere [6]. One finds that the real part of $I\left[T_{+}\right]$is:

$$
\operatorname{Re} I\left[T_{+}\right]=\frac{1}{2} \int_{-\infty}^{+\infty} d x^{-}\left(1-\cos \omega\left(x^{-}\right)\right) \partial_{-} v\left(x^{-}\right)
$$

It is the variation of the phase $v$ appearing in the non-diagonal elements of $R_{+}$ which plays the central role; $\operatorname{Re} I\left[T_{+}\right]=0$ if this phase is constant. 
One can compute $A_{-}$given by $-(i / g) \partial_{-} R_{+} R_{+}^{+}$. One gets a clumsy expression involving $\omega\left(x^{-}\right), v\left(x^{-}\right)$and their derivatives. The behaviour of $A_{-}$exhibits no distinctive feature if the path on the 2-sphere defined by $\left(\omega\left(x^{-}\right), v\left(x^{-}\right)\right)$ends at the south pole or goes through this point.

\section{Appendix B. Proving Lemma 4.1}

The proof of this lemma uses results of [11] but is more than a mere specialization of these results. For every $\bar{Q} \in \mathbf{Q}^{(-)}$it is immediate that $V$, as defined in (4.3), is an element of $\mathbf{M}$, with $V_{\infty}=1$. It has only vanishing right indices and is such that $V(x) \in S l(N, \mathbb{C})$. Therefore, as $G=S U(N)$, all we have to do to ensure that $V$ is in $V_{\|}$is to choose $\bar{Q}$ such that $V(x)$ is unitary.

We define a hermitian positive matrix function $B$ :

$$
B(x) \stackrel{\text { det }}{=} Q^{1}(x) Q(x) .
$$

$Q^{\dagger}(x)$ is the hermitian conjugate of the matrix $Q(x) ; B \in \mathbf{M}, B_{x}=1$. If $V(x)$ is unitary $B(x)$ is also given by

$$
B(x)=\bar{Q}^{\dagger}(x) \bar{Q}(x) .
$$

In the terminology of Grohberg and Krein [11], Eqs. (B.1) and (B.2) provide right and left standard factorizations of $B$ (the element of $\mathbf{M}^{(+)}$is on the right of the element of $\mathbf{M}^{(-)}$in (B.1), on its left in (B.2)). These factorizations contain no analogues of the central piece $m$ in (3.12). By its definition (B.1), $B$ has only vanishing right indices and all its left indices have to vanish too as a consequence of (B.2). This is automatic because if $B \in \mathbf{M}$, is hermitian and positive, all its right and left indices vanish and its right and left factorizations are unique [11]. Therefore $B$, which is determined by $Q$ via (B.1) defines a unique $\bar{Q}$ through (B.2).

\section{References}

1. Polyakov, A. M., Wiegmann, P. B.: Theory of nonabelian Goldstone bosons, in two dimensıons. Phys. Lett. 131B, 121-126 (1983)

2. Makowka, M., Wanders, G.: The determinant of massless fermions in two dimensions: A perturbative approach. Phys. Lett. 164B, 107-110 (1985);

Makowka, M., Wanders, G.: Perturbative derivation of the determinant of massless fermions in two dimensional space-time. Helv. Phys. Acta 59, 1366-1386 (1986)

3. Atiyah, M. F., Jones, J. D. S.: Topological aspects of Yang-Mills theory. Commun. Math. Phys. 61, 97-118 (1978); Alvarez-Gaumé, L., Ginsparg, P.: The topological meaning of non-abelian anomalies. Nucl. Phys. B243, 449-474 (1984)

4. Kupiainen, A., Mickelsson, J.. What is the effective action in two dimensions? Phys. Lett. 185B, $107-110(1987)$

5. DeWitt, B. S.: The space-time approach to quantum field theory. In: Relativity, groups and topology II (Les Houches 1983), pp. 381-738 DeWitt, B. S., Stora, R. (eds.). Amsterdam, Oxford, New York, Tokyo: North-Holland 1984; Yamagishi, H.: A space-time approach to chiral anomalies. Prog. Theor. Phys. 78, 886-907 (1987)

6. Cangemi, D., Makowka, M., Wanders, G.: The two dimensional Minkowski space fermion determinant by example. Phys. Lett. 211B, 107-110 (1988) 
7. Shale, D., Steinspring, W. F.: Spinor representations of infinite orthogonal groups. J. Math. Mech. 14, 315-322 (1965)

8. Labonté, G.: On the nature of "strong" Bogoliubov transfomations for fermions. Commun. Math. Phys. 36, 59-72 (1974)

9. Klaus, M., Sharf, G.: The regular external field problem in quantum electrodynamics. Helv. Phys. Acta 50, 779-802 (1977)

10. Wess, J., Zumino, B.: Consequences of anomalous Ward identities, Phys. Lett. 37B, 95-97 (1971); Witten, E.: Global aspects of current algebra. Nucl. Phys. B223, 422-444 (1983)

11. Gohberg, I. C., Krein, M. G.: Systems of integral equations on a half line with kernels depending on the difference of arguments. Am. Math. Soc. Transl. 2, 217-287 (1960); Litvinchuk, G. S., Spitkovski, I. M.: Factorization of measurable matrix functions. Basel: Birkhäuser 1987

12. Felder, G., Gawedzki, K., Kupiannen, A.: The spectrum of Wess-Zumino-Witten models. Nucl. Phys. B299, 355-366 (1988)

13. Pressley, L., Segal, G.: Loop groups. Oxford: Clarendon Press 1986

Communicated by K. Gawedzki

Received June 10, 1988; in revised form August 6, 1988 